\title{
Effects of Tea Polyphenols on Athletes
}

\author{
Junbiao Kang \\ School of Physical Education, Zhengzhou Institute of Technology, Zhengzhou 450000, China \\ tuke126@126.com \\ Corresponding Author: Junbiao Kang
}

Keywords: Tea Polyphenols; Tea; Strength; Antioxidant; Free Radical Scavenging.

\begin{abstract}
With the development of sports, it is urgent to find an object which can improve the athlete's strength and without any side effects. Studies have found that tea polyphenols (TP), with the effects of antioxidant and free radical scavenging, can improve the cardiac function and protect the integrity of mitochondria and other effects, which is an ideal nutritional supplements of athletes, with a strong development potential and application prospect.
\end{abstract}

\section{Introduction}

Tea polyphenol, also known as tea tannin, is a general name of all phenolic and phenolic derivatives in tea, with significant biological activity. There are more than 30 kinds of tea polyphenols which have been reported at present. Moreover, tea polyphenols are the most abundant substances, especially in green tea, accounting for about 35\%. While the content of catechin is the largest, accounting for more than one half. Modern pharmacological studies have shown that tea polyphenols are the main pharmacodynamic material basis in tea, which have remarkable antioxidant ability and free radical scavenging ability [1]. In the structure of catechins, the phenolic hydroxyl group in $\mathrm{B}$ ring and $\mathrm{C}$ ring has a strong ability to provide proton, which is reducing capacity, which is the material basis of tea polyphenols with strong oxidation capacity. In China, tea has a long history and far-reaching influence, as well as deep research and discussion. From the Classic of Tea in ancient time, people have begun the study of tea. Furthermore, tea is the homology of medicine and food. Therefore, tea polyphenols have been used in many ways. For example: (1) with the activity of free radical scavenging and antiviral; (2) to protect the bones by slowing down sports fatigue. The latter aspect is particularly significant in sports, which plays a great potential and good application prospects [2].

We will describe the effects of tea polyphenols from the following aspects:

The natural antioxidant are mainly found in many natural foods such as fruits, tea and Chinese herbal medicine, with significant antioxidant components such as the polyphenols, including flavonoids, anthocyanins, zine and selenium etc. Natural plant antioxidants act directly on a variety of free radicals associated with oxidative damage. On one hand, it can directly eliminate one or more free radicals simultaneously. On the other hand, it can inhibit the generation of free radicals, which plays a role in slowing down fatigue and anti-aging.

1. Improving the endurance of muscle

Modern research shows that catechin is a kind of natural antioxidant. As we all know, natural antioxidants have a strong ability in free radical scavenging, and also have a protective effect on cells $[3,4]$. Antioxidants can increase the biological activity of antioxidant enzymes in the blood of the body, and reduce the amount of free radicals. Tea polyphenols are the main active ingredients extracted from the tea. It is a kind of strong antioxidant which realize the antioxidation activity by scavenging free radical $[5,6]$. Tea polyphenols can not only increase the body's fat burning ability to improve the muscle endurance, but also provide the ability to fight fatigue so as to extent the physical exercise time. The more drink of green tea, the more significant effect of tea polypgenols [7]. Free radicals are generated during exercise. The free radicals beyond the normal level will break the balance of the body to make the body tired, thereby reducing the exercise ability. However, tea 
polyphenols, as the natural antioxidants, can reduce the role of free radicals through the interaction between active hydrogen in $\mathrm{B}$ ring and $\mathrm{C}$ ring and excessive free radicals, so that the body can be restored to the balance situation, and playing a role in slowing down fatigue. Moreover, the study on football player have proved that tea ployphenols extracted from the green tea can effectively reduce the content of serum oxide (such as serum malondialdehyde (MDA), serum urea, serum creatine kinase(CK)) produced after aerobic exercise, which suggests that tea polyphenols can reduce the free radicals during exercise, improve the body's antioxidant capacity, slow down fatigue and prolong the exercise time.

2. Protecting the biological activity of skeletal muscle

After intense exercise, calcium metabolism disorders will occur in the body's skeletal tissue, along with a large number of free radicals generated, so that skeletal muscle will be damaged easily. While the tea polyphenols can well regulate the concentration of calcium ion, inhibit the generate of calcium ion and maintain the balance of calcium ion in the body, so as to ensure the exchange between sudion and calcium ion in and out of cells, promote the normal metabolism of skeletal muscle, improve the energy exchange of skeletal muscle, and avoid the injury of skeletal muscle. At the same time, tea polyphenols can reduce the content of free radicals and dialdehyde produced by skeletal muscle during exercise, thereby reducing injury and improving exercise capacity from the aspects of antioxidant damage.

3. Protecting the biological activity of heart.

After intensive exercise, the free radicals content of myocardium will increase rapidly, leading to the enhanced signal pathway of downstream, which is likely to cause myocardial damage. Tea polyphenols in the tea can effectively reduce the free radicals produced after strenuous exercise, lessen the damage of myocardial issue, which play a role in protecting the myocardium. Tea polyphenols can also enhance the biological activity of cardiac function, promote the exercise tolerance and slow down fatigue.

4. Promoting the activity of nerve excitability

Nervous system, as the dominant of all human behaviors and activities, is an important regulation system of human body. Strenuous exercise will affect the function of neurons, resulting in the generation of a large number of metabolites and the deterioration of body environment, which will reduce the activity efficiency of neurons, thereby reducing the speed of information transmission. Meanwhile, people's reaction capacity and judgment ability will also be greatly affected, resulting in the impaired concentration and decreased movement strength, thereby reducing their exercise ability. Tea polyphenols can excite the central nervous system, regulate the metabolism of cerebral blood flow, speed up the exchange of calcium ions and stimulate the cerebral cortex, which will make people excited, enhance their memory, speed up the response ability and judgment, slow down the fatigue and enhance athletic ability [8].

5. Enhancing the function of oxygen transportation system

When doing exercise, $\mathrm{O}_{2}$ enters into the circulatory system, participates in the reaction, and produces the energy needed for exercise. The progress of aerobic exercise needs the decomposition of organic matter so as to provide the required energy, which requires the simultaneous participation of a large number of oxygen and organic matter to complete the supply of energy [9]. $\mathrm{O}_{2}$ is mainly transported with the combination of hemoglobin. Therefore, there is a close correlation between the content of hemoglobin and movement ability of human body. Studies have found that tea polyphenols can effectively promote the combination of $\mathrm{O}_{2}$ and hemoglobin, thus enhancing the body's transportation capacity of $\mathrm{O}_{2}$, which will avoid the fatigue caused by the cell hypoxia, and achieve the purpose of reducing fatigue and enhancing exercise capacity [10].

6. Effect of tea polyphenols on mitochondria

Mitochondria, as the "production workshop" of body's ability, is the source of energy. The anti-fatigue study of tea polyphenols on mouse have shown that tea ployphenols can enhance the exercise tolerance of the mouse, and strengthen the myocardial function. Adenosine Triphosphate (ATP) is the sole energy source of motion muscles, which is produced by oxidative phosphorylation of mitochondria. However, the increased free radicals in mitochondrial will affect the function of 
mitochondrial, resulting in the decreased ATP production, and thereby affecting the movement. While tea ployphenols can protect the mitochondria from damage, to ensure the integrity of motichondria, thus ensuring the normal muscle energy supply and enhancing the movement ability [11].

\section{Conclusion}

With the continuous development of sports in home and abroad, human beings are looking for the objects which can improve the movement ability of human body without any side effects. While tea polyphenols in tea can just meet these conditions. It not only can effectively remove the free radicals generated after exercise, playing a strong antioxidant, but also can protect the skeletal muscle and cardiac function, protect the integrity of mitochondria, and enhance the oxygen transport system and other biological activities. Studies have found that tea polyphenols can improve the exercise endurance of the body. Therefore, tea polyphenols has great potential and prospect not only in sports, but also in cancer prevention and human immunity improvement and other aspects of researches.

\section{References}

[1] Ge YZ, Jin H. A New Method for the Extraction of Tea-Polyphenols [J]. Chinese Traditional and Herbal Drugs. 1994, 25(3): 124-125.

[2] Sang HY, Wang L. Development and Utilization of Tea Polyphenols [J]. Science \& Technology Information. 2007, 24: 44-45.

[3] Li D. Six New Benefits of Drinking tea. Trends Health. 25-26.

[4] Kang K. Application and expectation of natural antioxidant in sports [J]. Journal of Beijing Sport University. 2000, 27(2), 19-21.

[5] Jiao HL, Ye P. Protective effects of green tea Polyphenols on human HePG2 cells against oxidative damage of fenofibrate [J]. Free Radical Biology Medicine, 2003, 35 (9): 1121-1128.

[6] Gao WB, Zhu YH, Wang YW. Effects of oral green tea polyphenols on the preservation of isolated rat heart [J]. The Journal of Practical Medicine. 2006, 22(12): 1362-1363.

[7] Deng GL, Ji LJ. Effect of Extract from Choerospondias Axillaries on Immunologic Function and Sports Endurance of Mice [J]. Journal of Sports\&Science. 2002, 23(5), 53-54.

[8] Wang WT. Study on Preparative Purification and Inhibiting Pseudomonas asruginosa Biofilm Properties of EGCG from Tea Polyphenols [D]. Jiangnan University, 2014.

[9] Luo LL, Li QG, Wang J. Impact and involving mechanisms of tea polyphenols on the biological behavior of A549 cell [J]. Jiangxi Medical Journal. 2015(9):886-890.

[10] Huang J. Biological Effects of Tea Polyphenols in Sports [J]. Tea \& Health. 2016, 2: 29-30.

[11] Shen SR, Jin CF, Chen ZY et al. Effects of Green Tea Polyphenols and Catechins on H2O2-induced Mitochondrial Permeability Transition Pore Opening [J]. Progress in Biochemistry and Biophysics. 2001, 28(6), 890-892. 